\title{
Coronary artery bypass grafting in a patient with pituitary adenoma: can alertness prevent tragedy?
}

\author{
Shitalkumar Shah ${ }^{1}$, FCARCSI, MRCA, Derek Hrabovsky², MBBS, FRCA
}

\begin{abstract}
Pituitary apoplexy is a rare, life-threatening complication that may occur after coronary artery bypass graft surgery for patients with pituitary adenomas. The dynamics of cardiopulmonary bypass may contribute to a sudden expansion of silent pituitary adenomas and result in the compression of surrounding structures. A range of clinical features have been described, and the condition requires prompt diagnosis and treatment to prevent further complications. Herein, we present an uncomplicated case highlighting the importance of diagnosing pituitary apoplexy, ensuring high alertness to the condition, so as to prevent life-threatening tragedy due to missed diagnosis.
\end{abstract}

Keywords: cardiopulmonary bypass, coronary artery bypass grafting, pituitary adenoma, pituitary apoplexy

\section{INTRODUCTION}

Pituitary macroadenomas, which affect 30 out of every 100,000 of the general population, ${ }^{(1)}$ are an infrequent cause of complications following cardiac surgery. Since the current literature contains few case reports of this complication, the most favourable treatment remains open-ended. (1) Pituitary apoplexy is a rare but life-threatening perioperative complication of coronary artery bypass graft (CABG) surgery. ${ }^{(2)}$ The clinical syndrome of pituitary apoplexy arises from: (a) unanticipated loss of pituitary hormonal secretion due to infarction of normal pituitary tissue; and (b) local anatomical compression by a briskly expanding pituitary tumour. The dynamics of cardiopulmonary bypass (CPB) contribute to the sudden expansion of silent pituitary adenomas. ${ }^{(3)}$ A range of clinical appearances for pituitary apoplexy has been described, including a lack of clinical symptoms, the presence of headache, a sudden decline in mental status, visual alteration, acute adrenal crisis (i.e. Addisonian crisis), ophthalmoplegia, and third cranial nerve palsy and/or ptosis. ${ }^{(2)}$ Pituitary apoplexy constitutes a critical neurosurgical emergency, and prompt diagnosis and treatment are vital to avoid further complications and terminal outcomes. ${ }^{(2)}$

Herein, we describe an uneventful and successful course of anaesthesia for CABG surgery in a patient with known preexisting pituitary macroadenoma. We also aim to highlight the importance of diagnosing pituitary apoplexy, ensuring alertness and maintaining a high index of suspicion to prevent a grave tragedy due to a missed diagnosis.

\section{CASE REPORT}

A 72-year-old man with a longstanding history of ischaemic heart disease presented with chest pain at rest. Coronary angiography revealed a recessive and diseased right coronary artery, and an ectatic left main stem coronary artery with a dominant and severely diseased circumflex coronary artery. The left ventricle function was good. On preanaesthetic assessment, a history of transient ischaemic attack occurring 40 years ago was described together with a recent diagnosis of pituitary macroadenoma.
During the course of investigating the patient's headaches, magnetic resonance (MR) imaging revealed the tumour, visualised as a well-defined mixed cystic and solid mass in the pituitary fossa. The macroadenoma measured $1.5 \mathrm{~cm} \times 1.3 \mathrm{~cm} \times 1.8 \mathrm{~cm}$, with the mass abutting the optic chiasm and both carotid arteries. An endocrinology review had initially reported a degree of hypotestosteronism, which had subsequently been resolved without medical treatment. At the time of surgery, prolactin, testosterone, thyroid function test, follicle-stimulating hormone and luteinising hormone levels were within their respective normal reference ranges. The patient was euthyroid, and there was no clinical evidence of acromegaly or goitre. In addition, the patient had a normal cortisol response to the short Synacthen test.

Based on the patient's stable pituitary macroadenoma status and in view of his coronary symptoms at rest, it was deemed best for surgery to proceed. Following invasive arterial blood pressure monitoring, right internal jugular central venous pressure (CVP) monitoring and pulmonary artery catheterisation, a preinduction intravenous (IV) 200-mg hydrocortisone bolus was administered. Induction commenced with a 2-mg midazolam bolus, targetcontrolled infusion of propofol at $1.5 \mu \mathrm{g} / \mathrm{ml}$, remifentanil infusion at $0.4 \mu \mathrm{g} / \mathrm{kg} / \mathrm{min}$, and a $10-\mathrm{mg}$ cisatracurium bolus. The patient underwent CABG surgery with $\mathrm{CPB}$ and core cooling to $34^{\circ} \mathrm{C}$. Clamp time was 33 mins, while pump time was 74 mins, allowing for two saphenous vein grafts. The operation was completed without any haemodynamic instability. Heparin was reversed using protamine sulphate, and the rate of return to baseline activated clotting time was measured. Perioperatively, mean arterial pressure was maintained at $65-70 \mathrm{mmHg}$ and CVP at 10-12 $\mathrm{mmHg}$, and the patient was extubated within a few hours following surgery. There was no sign of endocrine dysfunction, the patient's postoperative pituitary macroadenoma status remained stable, and his prolactin and thyroid function tests levels remained within normal ranges. On postoperative Day 2, he was transferred from the cardiac high dependency unit to the general ward with successful recovery. 


\section{DISCUSSION}

Pituitary apoplexy is a critical clinical syndrome, typically occurring due to a growing mass inside the sella turcica, arising from haemorrhage and/or necrosis or infarction within the tumour and neighbouring pituitary tissue. ${ }^{(2)}$ The growing mass could result in the compression of adjacent structures and the cranial nerves. Most vulnerable are the second, third, fourth, fifth and sixth cranial nerves, hypothalamus, cavernous and sphenoid sinuses and carotid artery. Signs and symptoms such as headache, visual disorders, ophthalmoplegia, ptosis, endocrinological abnormalities (e.g. hypothyroidism or Addisonian crisis), changes in mental status and even coma may be observed. ${ }^{(1,2,4-9)}$ Ophthalmoplegia may occur either without delay or up to several weeks following surgery. ${ }^{(2)}$ Total bilateral blindness is likely to occur if the pituitary adenoma is extensive, with major bleeding affecting both the optic nerves and the optic chiasma. ${ }^{(9)}$ Carotid artery occlusion may cause hemiparesis, and hypothalamic compression may cause sympathetic autoregulation changes. ${ }^{(3)}$

There are roughly 20 reported cases of pituitary apoplexy occurring after cardiac surgery..$^{(1,2,4-9)}$ One such case suggests patients undergo off-pump surgery to prevent pituitary apoplexy. ${ }^{(5)}$ However, it is uncertain what the root cause of postcardiac surgical pituitary apoplexy is. Numerous mechanisms during CPB, hypotension, hypoxia, cerebral hypoperfusion, anticoagulation, microembolism, and thrombosis may account for the condition. It is possible that patients with brittle and delicate pituitary adenoma tissues are extra vulnerable to perioperative ischaemic insult, ${ }^{(1,10,11)}$ which may lead to haemorrhage. Early diagnosis and effective intervention are critical for the successful management of pituitary apoplexy; a delay in management could result in fatal or irreversible neurological or endocrine damage. ${ }^{(12)}$

The differential diagnosis of pituitary apoplexy consists of undiagnosed intracranial tumour, ruptured intracranial aneurysm, and small vessel ischaemia. Computed tomography (CT) and MR imaging facilitate speedy diagnosis and can eliminate other intracranial disorders. Pituitary function tests are also central in guiding hormonal replacement therapy.(2) Transsphenoidal surgical intervention for timely decompression is the most common treatment option. ${ }^{(1,10,13,14)}$ Nevertheless, each individual case must be analysed carefully to assess the patient's anaesthesia and surgical stress. Vigilant assessment of coagulation status is also imperative, as intracranial bleeding may occur. To avoid this risk, conservative medical managements have also been successfully used in some cases of pituitary apoplexy. ${ }^{(8,9)}$ Most patients demonstrate complete recovery following timely surgery, ${ }^{(1,8,10,13,14)}$ and in most cases, perioperative hormonal replacement therapy is required.

In conclusion, this case report describes a positive outcome in a patient with known pituitary macroadenoma who underwent CABG surgery despite CPB. In this context, we sought a full preoperative endocrine workup to confirm that the patient's pituitary macroadenoma status was stable and minimise any potential risks. Irrespective of the preoperative preparation, we ensured that the patient was fully informed of the risks of pituitary haemorrhage, infarction and subsequent complications consequences of CABG surgery. Meticulous attention to and monitoring of haemodynamic parameters, and a full reversal of anticoagulation with thromboelastography and activated clotting time were instituted throughout the perioperative period. In the case of our patient, the outcome was favourable.

\section{REFERENCES}

1. Pliam MB, Cohen M, Cheng L, et al. Pituitary adenomas complicating cardiac surgery: Summary and review of 11 cases. J Card Surg 1995; 10:125-32.

2. Chen Z, Murray AW, Quinlan JJ. Pituitary apoplexy presenting as unilateral third cranial nerve palsy after coronary artery bypass surgery. Anesth Analg 2004; 98:46-8.

3. Hidiroglu M, Kucuker A, Ucaroglu E, Kucuker SA, Sener E. Pituitary apoplexy after cardiac surgery. Ann Thorac Surg 2010; 89:1635-7.

4. Meek EN, Butterworth J, Kon ND, et al. Pituitary apoplexy following mitral valve repair. Anesthesiology 1998; 89:1580-2.

5. Levy E, Korach A, Merin G, Feinsod M, Glenville B. Pituitary apoplexy and CABG: should we change our strategy? Ann Thorac Surg 2007; 84:1388-90.

6. Mayrin JV, Laufgraben M. Pituitary apoplexy following cardiac surgery: a case report and review of the literature. Endocrinologist 2007; 17: 214-6.

7. Aksoy T, Karaca P, Atasoy MM, Sahin S. Isolated third-nerve palsy after cardiac surgery. J Cardiothorac Vasc Anesth 2007; 21:110-2.

8. Alzetani A, Fisher C, Costa R, Ohri SK. Ptosis postcardiac surgery: a case of pituitary apoplexy. Ann Thorac Surg 2002; 73:300-1.

9. Loubani M, Galinanes M. Pituitary gland macroadenoma: a cause of transient blindness after cardiac surgery. Ann Thorac Surg 2001; 72:929-31.

10. Mattke AF, Vender JR, Anstadt MR. Pituitary apoplexy presenting as Addisonian crisis after coronary artery bypass grafting. Tex Heart Inst J 2002; 29:193-9.

11. Kovacs K, Yao J. Pituitary necrosis following major heart surgery. Z Kardiol 1975; 64:52-7.

12. Randeva H, Schoebel J, Byrne J, et al. Classical pituitary apoplexy: clinical features, management and outcome. Clin Endocrinol (Oxf)1999; 51:181-8.

13. Kontorinis N, Holthouse DJ, Carroll WM, Newman M. Third nerve palsy after coronary artery bypass surgery. J Thorac Cardiovasc Surg 2001; 122:400-1.

14. Wiesmann M, Gliemroth J, Kehler U, Missler U. Pituitary apoplexy after cardiac surgery presenting as deep coma with dilated pupils. Acta Anaesthesiol Scand 1999; 43:236-8. 